\title{
Phase-dependent magnetoconductance fluctuations in a chaotic Josephson junction
}

\author{
P. W. Brouwer and C. W. J. Beenakker \\ Instituut-Lorentz, University of Leiden, P.O. Box 9506, 2300 RA Leiden, The Netherlands
}

(Received 9 July 1996)

\begin{abstract}
Motivated by recent experiments by Den Hartog et al, we present a random-matrix theory for the magnetoconductance fluctuations of a chaotic quantum dot that is coupled by point contacts to two superconductors and one or two normal metals. There are aperiodic conductance fluctuations as a function of the magnetic field through the quantum dot and $2 \pi$-periodic fluctuations as a function of the phase difference $\phi$ of the superconductors. If the coupling to the superconductors is weak compared to the coupling to the normal metals, the $\phi$ dependence of the conductance is harmonic, as observed in the experiment. In the opposite regime, the conductance becomes a random $2 \pi$-periodic function of $\phi$, in agreement with the theory of Altshuler and Spivak. The theoretical method employs an extension of the circular ensemble which can describe the magnetic-field dependence of the scattering matrix. [S0163-1829(96)51442-6]
\end{abstract}

The conductance of a mesoscopic metal shows small fluctuations of universal size $e^{2} / h$ as a function of magnetic field.' These universal conductance fluctuations are sample specific, which is why a plot of conductance $G$ versus magnetic field $B$ is called a "magnetofingerprint." The magnetoconductance is sample specific because it depends sensitively on scattering phase shifts, and hence on the precise configuration of scatterers. Any agency which modifies phase shifts will modify the magnetoconductance. Altshuler and Spivak ${ }^{2}$ first proposed to use a Josephson junction for this purpose. If the metal is connected to two superconductors with a phase difference $\phi$ of the order parameter, the conductance $G(B, \phi)$ contains two types of sample-specific fluctuations: aperiodic fluctuations as a function of $B$ and $2 \pi$-periodic fluctuations as a function of $\phi$. The magnetic field should be sufficiently large to break time-reversal symmetry, otherwise the sample-specific fluctuations will be obscured by a much stronger $B$ and $\phi$ dependence of the ensemble-averaged conductance. ${ }^{3-5}$

In a recent paper, den Hartog et $a l .{ }^{6}$ reported the experimental observation of phase-dependent magnetoconductance fluctuations in a $T$-shaped two-dimensional electron gas. The horizontal arm of the $T$ is connected to two superconductors, the vertical arm to a normal metal reservoir. The observed magnitude of the fluctuations was much smaller than $e^{2} / h$, presumably because the motion in the $T$ junction was nearly ballistic. Larger fluctuations are expected if the arms of the $T$ are closed, leaving only a small opening (a point contact) for electrons to enter or leave the junction. Motion in the junction can be ballistic or diffusive, as long as it is chaotic the statistics of the conductance fluctuations will only depend on the number of modes in the point contacts and not on the microscopic details of the junction.

In this paper we present a theory for phase-dependent magnetoconductance fluctuations in a chaotic Josephson junction. We distinguish two regimes, depending on the relative magnitude of the number of modes $M$ and $N$ in the point contacts to the superconductors and normal metals, respectively. For $M \gg N$ the $\phi$ dependence of the conductance is strongly anharmonic. This is the regime studied by Altshuler and Spivak. ${ }^{2}$ For $M \leqq N$ the oscillations are nearly sinu- soidal, as observed by den Hartog et al. ${ }^{6}$ The difference between the two regimes can be understood qualitatively in terms of interfering Feynman paths. In the regime $M \leq N$ only paths with a single Andreev reflection contribute to the conductance. Each such path depends on $\phi$ with a phase factor $e^{ \pm \imath \phi / 2}$. Interference of these paths yields a sinusoidal $\phi$ dependence of the conductance. In the opposite regime $M \gg N$, quasiparticles undergo many Andreev reflections before leaving the junction. Hence higher harmonics appear, and the conductance becomes a random $2 \pi$-periodic function of $\phi$.

The system under consideration is shown schematically in Fig. 1. It consists of a chaotic cavity in a time-reversalsymmetry breaking magnetic field $B$, which is coupled to two superconductors and to one or two normal metals by ballistic point contacts. The superconductors $\left(S_{1}\right.$ and $\left.S_{2}\right)$ have the same voltage (defined as zero) and a phase difference $\phi$. The conductance of this Josephson junction is measured in a three- or four-terminal configuration. In the threeterminal configuration (Fig. 1a), a current $I$ flows from a normal metal $N_{1}$ into the superconductors. The conductance $G=I / V_{1}$ is the ratio of $I$ and the voltage difference $V_{1}$ between $N_{1}$ and $S_{1}, S_{2}$. This corresponds to the experiment of den Hartog et al. ${ }^{6}$ In the four-terminal configuration (Fig. 1b), a current $I$ flows from a normal metal $N_{1}$ into another metal $N_{2}$. The conductance $G=I /\left(V_{1}-V_{2}\right)$ now contains the voltage difference between $N_{1}$ and $N_{2}$. This is the configuration studied by Altshuler and Spivak. ${ }^{2}$

Following Ref. 6 we split the conductance $G(B, \phi)$ $=G_{0}(B)+G_{\phi}(B, \phi)$ into a $\phi$-independent background

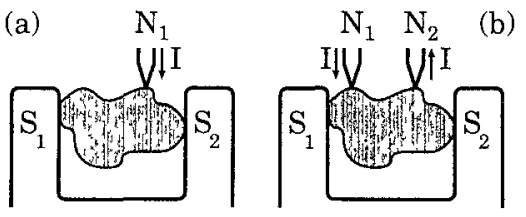

FIG. 1. Josephson junction in a three-terminal (a) and fourterminal (b) configuration. 


$$
G_{0}(B)=\int_{0}^{2 \pi} \frac{d \phi}{2 \pi} G(B, \phi),
$$

plus $2 \pi$-periodic fluctuations $G_{\phi}$. In the absence of timereversal symmetry, the ensemble average $\langle G(B, \phi)\rangle \equiv\langle G\rangle$ is independent of $B$ and $\phi$. Hence $\left\langle G_{0}(B)\right\rangle=\langle G\rangle$ and $\left\langle G_{\phi}(B, \phi)\right\rangle=0$. The correlator of $G$ is

$$
C(\delta B, \delta \phi)=\langle G(B, \phi) G(B+\delta B, \phi+\delta \phi)\rangle-\langle G\rangle^{2} .
$$

Fluctuations of the background conductance are described by the correlator of $G_{0}$,

$$
\begin{aligned}
C_{0}(\delta B) & =\left\langle G_{0}(B) G_{0}(B+\delta B)\right\rangle-\langle G\rangle^{2} \\
& =\int_{0}^{2 \pi} \frac{d \delta \phi}{2 \pi} C(\delta B, \delta \phi) .
\end{aligned}
$$

(In the second equality we used that $\left\langle G_{\phi} G_{0}\right\rangle=0$.) The difference $C_{\phi}=C-C_{0}$ is the correlator of $G_{\phi}$,

$$
C_{\phi}(\delta B, \delta \phi)=\left\langle G_{\phi}(B, \phi) G_{\phi}(B+\delta B, \phi+\delta \phi)\right\rangle .
$$

We compute these correlators for the three- and fourterminal configurations, beginning with the former.

In the three-terminal configuration, the cavity is connected to three point contacts (Fig. 1a). The contact to the normal metal has $N$ propagating modes at the Fermi energy and the contacts to the superconductors have $M / 2$ modes each. The $(N+M) \times(N+M)$ scattering matrix $S$ of the cavity is decomposed into $M \times M(N \times N)$ reflection matrices $r\left(r^{\prime}\right)$ and $N \times M(M \times N)$ transmission matrices $t\left(t^{\prime}\right)$,

$$
S=\left(\begin{array}{ll}
r & t^{\prime} \\
t & r^{\prime}
\end{array}\right)
$$

The conductance at zero temperature is determined by the matrix $s_{h e}$ of scattering amplitudes from electron to hole, ${ }^{7-9}$

$$
\begin{gathered}
G=2 \operatorname{tr} s_{h e} s_{h e}^{\dagger}, \\
s_{h e}=-i t^{*}\left(1+e^{i \Phi} r e^{-i \Phi} r^{*}\right)^{-1} e^{i \Phi} t^{\prime} .
\end{gathered}
$$

The diagonal matrix $\Phi$ has diagonal elements $\Phi_{n n}=\phi / 2$ if $1 \leqslant n \leqslant M / 2$ and $-\phi / 2$ if $1+M / 2 \leqslant n \leqslant M$. We measure $G$ in units of $2 e^{2} / h$.

For chaotic scattering without time-reversal symmetry, the matrix $S$ is uniformly distributed in the unitary group. ${ }^{10}$ This is the circular unitary ensemble (CUE) of randommatrix theory. ${ }^{11}$ The CUE does not specify how $S$ at different values of $B$ is correlated. The technical innovation used in this work is an extension of the CUE, which includes the parametric dependence of the scattering matrix on the magnetic field. The method (described in detail elsewhere ${ }^{12}$ ) consists in replacing the magnetic field by a time-reversalsymmetry breaking stub (see Fig. 2). This idea is similar in spirit to Büttiker's method of modeling inelastic scattering by a phase-breaking lead. ${ }^{13}$ The stub contains $N_{\text {stub }}$ modes. The end of the stub is closed, so that it conserves the number of particles without breaking phase coherence. (Büttiker's lead, in contrast, is attached to a reservoir, which conserves the number of particles by matching currents, not amplitudes, and therefore breaks phase coherence.) We choose our

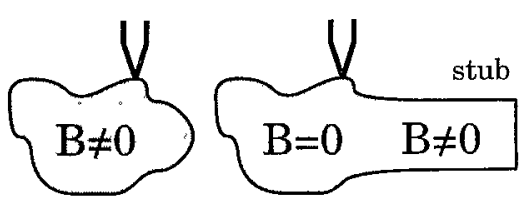

FIG. 2. Schematic picture how the magnetic field is included in the scattering-matrix ensemble. A chaotic cavity with a spatially homogeneous magnetic field (left diagram) is statistically equivalent to a chaotic cavity in zero magnetic field (right diagram), which is coupled to a closed lead (a stub) having a nonsymmetric reflection matrix.

scattering basis such that the $N_{\text {stub }} \times N_{\text {stub }}$ reflection matrix $r_{\text {stub }}(B)$ of the stub equals the unit matrix at $B=0$. For nonzero magnetic fields we take

$$
r_{\text {stub }}(B)=e^{B A}, \quad a^{2} \equiv \sum_{n<m} A_{n m}^{2},
$$

where the matrix $A$ is real and antisymmetric: $A_{n m}=A_{n m}^{*}=-A_{m n}$. Particle number is conserved by the stub because $r_{\text {stub }}$ is unitary, but time-reversal symmetry is broken because $r_{\text {stub }}$ is not symmetric if $B \neq 0$. In order to model a spatially homogeneous magnetic field, it is essential that $N_{\text {stub }} \gg N+M$. The value of $N_{\text {stub }}$ and the precise choice of $A$ are irrelevant, all results depending only on the single parameter $a$.

The magnetic-field dependent scattering matrix $S(B)$ in this model takes the form

$$
S(B)=U_{11}+U_{12}\left[1-r_{\text {stub }}(B) U_{22}\right]^{-1} r_{\text {stub }}(B) U_{21} .
$$

The matrices $U_{i j}$ are the four blocks of a matrix $U$ representing the scattering matrix of the cavity at $B=0$, with the stub replaced by a regular lead. The distribution of $U$ is the circular orthogonal ensemble (COE), which is the ensemble of uniformly distributed, unitary and symmetric matrices. ${ }^{11}$ The distribution of $S(B)$ resulting from Eqs. (7) and (8) crosses over from the COE for $B=0$ to the CUE for $B \rightarrow \infty$. One can show ${ }^{12}$ that it is equivalent to the distribution of scattering matrices following from the Pandey-Mehta Hamiltonian ${ }^{14}$ $H=H_{0}+i B H_{1}$ [where $H_{0}\left(H_{1}\right)$ is a real symmetric (antisymmetric) Gaussian distributed matrix].

It remains to relate the parameter $a$ to microscopic properties of the cavity. We do this by computing the correlator $\Sigma_{m n}(\delta B)=\left\langle S_{m n}(B) S_{m n}^{*}(B+\delta B)\right\rangle$ from Eq. (8). Using the diagrammatic method of Ref. 15 to perform the average over the $\mathrm{COE}$, we find (for $N+M \gg 1$ )

$$
\Sigma_{m n}=(N+M)^{-1}\left[1+\left(\delta B / B_{c}\right)^{2}\right]^{-1}, \quad n \neq m,
$$

with $B_{c} \equiv a^{-1} \sqrt{N+M}$. This correlator of scattering matrix elements also has been computed by other methods. ${ }^{16-19}$ Comparing results we can identify

$$
a^{2}=c e^{2} v_{F} L^{2} \min (\ell, L) / \hbar \delta,
$$

with $c$ a numerical coefficient of order unity depending on the shape of the cavity (linear dimension $L$, mean free path $\ell$, Fermi velocity $v_{F}$, and level spacing $\delta$ ). For example, for a disordered disk or sphere (radius $L \gg \ell$ ) the coefficient $c=\pi / 8$ for the disk and $\pi / 15$ for the sphere. 
We now proceed with the calculation of the correlator of the conductance. We consider broken time-reversal symmetry $\left(B \gg B_{c}\right)$ and assume that $N$ and $M$ are both $\gg 1$. Using the method of Ref. 15 for the average over $U$, we obtain the average conductance $\langle G\rangle=2 N M /(N+2 M)$ and the correlator

$$
\begin{aligned}
C(\delta B, \delta \phi)= & 16 K N^{2} M^{2}(N+M)^{2}(N+2 M)^{-4} \\
& \times \frac{(N+M)^{2}+\left(N^{2}+M^{2} K\right) \cos ^{2}(\delta \phi / 2)}{\left[(N+M)^{2}-M^{2} K \cos ^{2}(\delta \phi / 2)\right]^{2}},
\end{aligned}
$$

where we have abbreviated $K=\left[1+\left(\delta B / B_{c}\right)^{2}\right]^{-2}$. Equation (11) simplifies considerably in the two limiting regimes $M \ll N$ and $M \gg N$. For $M \ll N$ we find

$$
\begin{gathered}
C_{0}(\delta B)=24(M / N)^{2} K \\
C_{\phi}(\delta B, \delta \phi)=8(M / N)^{2} K \cos \delta \phi
\end{gathered}
$$

whereas for $M \gg N$ we have (for $|\delta \phi|<\pi$ )

$$
\begin{aligned}
C_{0}(\delta B) & =\sqrt{\frac{N}{8 M}}\left[1+\frac{M}{N}\left(\frac{\delta B}{B_{c}}\right)^{2}\right]^{-3 / 2} \\
C_{\phi}(\delta B, \delta \phi) & =\frac{1}{2}\left[1+\frac{M}{N}\left(\frac{\delta B}{B_{c}}\right)^{2}+\frac{M}{8 N} \delta \phi^{2}\right]^{-2}
\end{aligned}
$$

The two regimes differ markedly in several respects:

(1) The $2 \pi$-periodic conductance fluctuations are harmonic if $M \ll N$ and highly anharmonic if $M \gg N$. A small increment $\delta \phi \simeq \sqrt{N / M} \ll 2 \pi$ of the phase difference between the superconducting contacts is sufficient to decorrelate the conductance if $M \gg N$.

(2) The variance of the conductance $\operatorname{var} G$ $=C_{0}(0)+C_{\phi}(0,0)$ has the universal magnitude $1 / 2$ if $M \gg N$, while it is reduced by a factor $(8 M / N)^{2}$ if $M \ll N$.

(3) The variance $\operatorname{var} G_{\phi}=C_{\phi}(0,0)$ of the $\phi$-dependent conductance is larger than the variance $\operatorname{var} G_{0}=C_{0}(0)$ of the background conductance if $M \gg N$ (by a factor $\sqrt{M / 8 N}$ ), while it is smaller if $M \ll N$ (by a factor $1 / 3$ ).

(4) The correlators $C_{\phi}(\delta B, 0)$ and $C_{0}(\delta B)$ both decay as a squared Lorentzian in $\delta B / B_{c}$ if $M \ll N$. If $M \gg N$, on the contrary, $C_{\phi}(\delta B, 0)$ decays as a squared Lorentzian, while $C_{0}(\delta B)$ decays as a Lorentzian to the power $3 / 2$.

The difference between the two limiting regimes is illustrated in Fig. 3. The "sample-specific" curves in the upper panels were computed from Eq. (6) for a matrix $S$ which was randomly drawn from the CUE. The correlators in the lower panels were computed from Eq. (11). The qualitative difference between $M \leq N$ (Fig. 3a) and $M \gg N$ (Fig. 3b) is clearly visible.

We now turn to the four-terminal configuration (Fig. 1b). The two point contacts to the superconductors have $M / 2$ modes each, as before; The two point contacts to the normal metals have $N / 2$ modes each. The conductance is given by the four-terminal generalization of Eq. (6), ${ }^{7}$

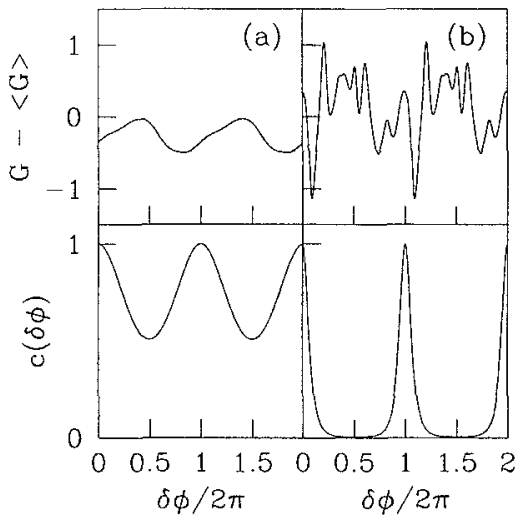

FIG. 3. Top panels: conductance minus the ensemble average (in units of $2 e^{2} / h$ ) as a function of the phase difference between the superconductors. Bottom panels: normalized correlator $c(\delta \phi)=C(0, \delta \phi) / C(0,0)$, computed from Eq. (11). (a) is for $N=120, M=60$; (b) is for $N=10, M=160$.

$$
\begin{gathered}
G=R_{21}^{e e}+R_{21}^{h e}+\frac{2\left(R_{11}^{h e} R_{22}^{h e}-R_{12}^{h e} R_{21}^{h e}\right)}{R_{11}^{h e}+R_{22}^{h e}+R_{12}^{h e}+R_{21}^{h e}}, \\
R_{i j}^{h e}=\operatorname{tr} s_{h e} c_{j} s_{h e}^{\dagger} c_{i}, \quad R_{i j}^{e e}=\operatorname{tr} s_{e e c_{j} s_{e e}^{\dagger} c_{i},} \\
s_{e e}=r^{\prime}-t e^{-i \Phi} r^{*}\left(1+e^{i \Phi} r e^{-i \Phi} r^{*}\right)^{-1} e^{i \Phi} t^{\prime} .
\end{gathered}
$$

Here $\left(c_{1}\right)_{m n}=1$ if $m=n \leqslant N / 2$ and 0 otherwise, and $c_{2}=1-c_{1}$. The matrix $s_{h e}$ was defined in Eq. (6b). Performing the averages as before, we find $\langle G\rangle=N / 4$ and

$$
\begin{aligned}
C(\delta B, \delta \phi)= & \frac{1}{16} N^{2} K\left[(N+M)^{2}+M^{2} K \cos ^{2}(\delta \phi / 2)\right] \\
& \times\left[(N+M)^{2}-M^{2} K \cos ^{2}(\delta \phi / 2)\right]^{-2}
\end{aligned}
$$

In the regime $M \ll N$ this simplifies to

$$
\begin{gathered}
C_{0}(\delta B)=\frac{1}{16} K \\
C_{\phi}(\delta B, \delta \phi)=\frac{3}{32}(M / N)^{2} K^{2} \cos \delta \phi
\end{gathered}
$$

while in the regime $M \gg N$ we find again Eq. (13) (with an extra factor of $1 / 16$ on the r.h.s.).

The four-terminal configuration with $M \gg N$ is similar to the system studied by Altshuler and Spivak. ${ }^{2}$ One basic difference is that they consider the high-temperature regime $k_{B} T \gg \hbar / \tau_{\text {dwell }}$ (with $\tau_{\text {dwell }}$ the mean dwell time of a quasiparticle in the junction), while we assume $T=0$ (which in practice means $k_{B} T \ll \hbar / \tau_{\text {dwell }}$ ). Because of this difference in temperature regimes, we cannot make a detailed comparison with the results of Ref. 2 .

The features of the regime $M \leqq N$ in the three-terminal configuration agree qualitatively with the experimental observations made by den Hartog et al. ${ }^{6}$ In particular, they find a nearly sinusoidal $\phi$ dependence of the conductance, with $C_{\phi}(B, 0)$ being smaller than $C_{0}(B)$, while having the same $B$ dependence. The magnitude of the fluctuations which they observe is much smaller than what we find for a pointcontact coupling with $M$ and $N$ of comparable magnitude. This brings us to the prediction that the insertion of a point contact in the vertical arm of the $T$ junction of Ref. 6 (which is connected to a normal metal) would have the effect of (1) 
increasing the magnitude of the magnetoconductance fluctuations so that it would become of order $e^{2} / h$, (2) introducing higher harmonics in the $\phi$ dependence of the conductance This should be a feasible experiment which would probe an interestıng new regime

In conclusion, we have calcuiated the correlation function of the conductance of a chaotic cavity coupled via point contacts to two superconductors and one or two normal metals, as a function of the magnetic field through the cavity and the phase difference between the superconductors If the superconducting point contacts dominate the conductance, the phase-dependent conductance fluctuations are harmonic, whereas they become highly anharmonic if the normal point contact limits the conductance The harmonic regime has been observed in Ref 6 , and we have suggested a modifica-

'B L Altshuler, Pis'ma Zh Eksp Teor F1Z 41, 530 (1985) [JETP Lett 41, 648 (1985)], P A Lee and A D Stone, Phys Rev Lett 55, 1622 (1985)

${ }^{2}$ B L Altshuler and B Z Spivak, Zh Éksp Teor Fiz 92, 609 (1987) [Sov Phys JETP 65, 343 (1987)]

${ }^{3}$ For a review, see C W J Beenakker, in Mesoscopic Quantum Physics, edited by E Akkermans, G Montambaux, J -L P1chard, and J Zinn-Justun (North-Holland, Amsterdam, 1995)

${ }^{4}$ Sample-specific conductance fluctuations at zero magnetıc field have been observed experımentally by $\mathrm{P} G \mathrm{~N}$ de Vegvar, $\mathrm{T}$ A Fulton, W H Mallison, and R E Miller, Phys Rev Lett 73, 1416 (1994)

${ }^{5}$ In zero magnetic field and at sufficiently high temperatures, the average conductance is a $\pi$-periodic function of $\phi$, see $B Z$ Spivak and D E Khmelnitski1, Pis'md Zh Eksp Teor Fiz 35, 334 (1982) [JETP Lett 35, 412 (1982)] In the absence of timereversal symmetry the periodicity is $2 \pi$

${ }^{6} \mathrm{~S}$ G den Hartog, C M A Kapteyn, B J van Wees, T M tion of the experiment to probe the anhatmonic regime as well We introduced a novel technique to compute the magnetoconductance fluctuations, consisting in the replacement of the magnetic field by a time-reversal-symmetry breaking stub This extension of the crrcular ensemble is likely to be useful in other applications of random-matrix theory to mesoscopic systems

We have benefited from discussions with the participants of the workshop on "Quantum Chaos" at the Institute for Theoretical Physics in Santa Barbara, in particular with B L Altshuler Discussions with B $\mathbf{J}$ van Wees are also giatefully acknowledged This work was supported in part by the Dutch Science Foundation NWO/FOM and by the NSF under Grant No PHY94-07194

Klapwijk, W van der Graaf, and G Borghs Phys Rev Lett 76, 4592 (1996)

${ }^{7}$ C J Lambert, J Phys, Condens Matter 3, 6579 (1991)

${ }^{8} \mathrm{Y}$ Takane and H Ebisawa, J Phys Soc Jpn 60, 3130 (1991)

${ }^{9} \mathrm{C}$ W J Beenakker, Phys Rev B 46, 12841 (1992)

${ }^{10} \mathrm{R}$ Blumel and U Smılansky, Phys Rev Lett 60, 477 (1988)

1 M L Mehta, Random Matrices (Academic, New York, 1991)

12 P W Brouwer (unpublished)

${ }^{13}$ M Buttıker, Phys Rev B 33, 3020 (1986)

${ }^{14}$ A Pandey and M L Mehta, Commun Math Phys 87, 449 (1983)

${ }^{15} \mathrm{P} \mathrm{W}$ Brouwer and C W J Beenakker (unpublished)

${ }^{16} \mathrm{R}$ A Jalabert, H U Baranger, and A D Stone, Phys Rev Lett 65, $1442(1990)$

${ }^{17}$ Z Pluhař, H A Weidenmuller, J A Zuk, and C H Lewenkopf, Phys Rev Lett 73, 2115 (1994)

${ }^{18}$ K B Efetov, Phys Rev Lett 74, 2299 (1995)

${ }^{19}$ K Frahm, Europhys Lett 30, 457 (1995) 